\title{
Intraperitoneal Chemotherapy of Peritoneal Carcinomatosis Using Pressurized Aerosol as an Alternative to Liquid Solution: First Evidence for Efficacy
}

\author{
Wiebke Solass, $\mathrm{MD}^{1}$, Reinhold Kerb, $\mathrm{MD}^{2,3}$, Thomas Mürdter, $\mathrm{PhD}^{2,3}$, Urs Giger-Pabst, $\mathrm{MD}^{4}$, Dirk Strumberg, \\ $\mathrm{MD}^{5}$, Clemens Tempfer, MD, MBA ${ }^{6}$, Jürgen Zieren, $\mathrm{MD}^{4}$, Matthias Schwab, $\mathrm{MD}^{2,3}$, and Marc André Reymond, \\ $\mathrm{MD}, \mathrm{MBA}^{4}$
}

${ }^{1}$ Institute of Pathology, Ruhr-University Bochum, Bochum, Germany; ${ }^{2}$ Dr. Margarete Fischer-Bosch Institute of Clinical Pharmacology, Stuttgart, Germany; ${ }^{3}$ Department of Clinical Pharmacology, University Hospital, Tübingen, Germany; ${ }^{4}$ Department of Surgery, Marienhospital Herne, Ruhr-University Bochum, Bochum, Germany; ${ }^{5}$ Department of Internal Medicine, Oncology and Hematology, Marienhospital Herne, Ruhr-University Bochum, Bochum, Germany; ${ }^{6}$ Department of Gynaecology and Obstetrics, Marienhospital Herne, Ruhr-University Bochum, Bochum, Germany

\begin{abstract}
Background. Peritoneal carcinomatosis (PC) is an unmet medical need. Despite recent improvements, systemic chemotherapy has limited efficacy. We report the first application of intraperitoneal chemotherapy as a pressurized aerosol in human patients.

Methods. Three end-stage patients with advanced PC from gastric, appendiceal, and ovarian origin were treated as a compassionate therapy. All patients had received previous systemic chemotherapy. A pressurized aerosol of $\mathrm{CO}_{2}$ loaded with doxorubicin $1.5 \mathrm{mg} / \mathrm{m}^{2}$ and cisplatin $7.5 \mathrm{mg} / \mathrm{m}^{2}$ (pressurized intraperitoneal aerosol chemotherapy, PIPAC) was applied into the abdomen for $30 \mathrm{~min}$ at a pressure of $12 \mathrm{mmHg}$ and a temperature of $37^{\circ} \mathrm{C}$.

Results. No side-effects $>2$ CTCAE were observed, and the procedures were well tolerated. Early hospital discharge was possible (days 2-5). Nuclear presence of doxorubicin was documented throughout the peritoneum, reaching high local concentration $(\leq 4.1 \mu \mathrm{mol} / \mathrm{g})$ and plasma concentration was low $(4.0-6.2 \mathrm{ng} / \mathrm{ml})$. PIPAC
\end{abstract}

Electronic supplementary material The online version of this article (doi:10.1245/s10434-013-3213-1) contains supplementary material, which is available to authorized users.

(C) The Author(s) 2013. This article is published with open access at Springerlink.com

First Received: 23 April 2013;

Published Online: 5 September 2013

M. A. Reymond, MD, MBA

e-mail: marc.reymond@yahoo.de created no significant adhesions, could be repeated, and was applied $6 \times, 4 \times$, and $2 \times$. Two patients showed a complete and one a partial histological remission. Mean survival after the first PIPAC was 288 days. One patient is alive after 567 days.

Conclusions. PIPAC shows superior pharmacological properties with high local concentration and low systemic exposure. PIPAC can induce regression of PC in chemoresistant tumors, using $10 \%$ of a usual systemic dose.

Life expectancy in peritoneal carcinomatosis (PC) is limited due to advanced tumor stage and poor therapeutic response. Resistance of PC to systemic chemotherapy (SC) is explained by molecular mechanisms and by limited drug distribution. ${ }^{1,2}$ This is the rationale for locoregional therapy combining cytoreductive surgery (CRS) with intraperitoneal chemotherapy (IPC). ${ }^{3}$ However, this approach is debated: IPC is hampered by limited drug distribution within the abdominal cavity and poor penetration into PC nodules. ${ }^{4,5}$ Thus, the benefit of combined CRS and IPC compared with CRS alone might be marginal. ${ }^{6}$

We have proposed to apply chemotherapy as a pressurized aerosol within the abdominal cavity to take advantage of following physical properties: applying an aerosol allows a homogeneous repartition of the substance within a closed space; generating an artificial pressure gradient counterbalances tumoral interstitial fluid pressure, an obstacle in cancer therapy. ${ }^{7,8}$ In the large animal model, pressurized aerosol improved both distribution of a vital staining within the abdominal cavity, and depth of 
penetration into the peritoneum, as compared to peritoneal lavage with a liquid solution. ${ }^{9}$ When treating human PC ex vivo, we achieved a superior distribution onto the peritoneum and a better penetration into PC nodules than IPC. ${ }^{10}$ This was the rationale for the first application in the human patient.

\section{METHODS}

\section{Patients}

Pressurized intraperitoneal aerosol chemotherapy (PIPAC) was offered as a treatment option to three patients suffering from a fatal disease for which no satisfactory alternative therapy was available, pursuant to the individual compassionate use of medicinal products according to the German Medical Act (AMG) and with documented favorable opinion by the Ethics Committees of the University of Münster, Germany. Patients were evaluated by our multidisciplinary team before onset of treatment and provided written, informed consent. Clinical and histological confirmation of PC, including small bowel involvement, was required. No patient had parenchymatous metastases. Patient histories are summarized in Table 1. PIPACs were performed between November 2011 and March 2013.

\section{Surgical Procedures}

PIPAC is described in Fig. 1. After insufflation of a $12 \mathrm{mmHg}$ of capnoperitoneum at $37^{\circ} \mathrm{C}$, two balloon trocars (Applied Medical, Düsseldorf) were placed. Explorative laparoscopy was performed as usual and PC index was determined. ${ }^{11}$ Parietal biopsies were taken and ascites was removed. A nebulizer (MIP, Reger Medizintechnik, Rottweil) was connected to a high-pressure injector (Injektron 82M, MedTron, Saarbruecken) and inserted into the abdomen through a trocar. A pressurized aerosol containing cisplatin (Hexal, Barleben) at a dose of $7.5 \mathrm{mg} / \mathrm{m}^{2}$ body surface in $150 \mathrm{ml} \mathrm{NaCl} 0.9 \%$ was applied immediately followed by doxorubicin (Hexal, Barleben) $1.5 \mathrm{mg} / \mathrm{m}^{2}$ in $50 \mathrm{ml} \mathrm{NaCl} 0.9 \%$. Then, the system was kept in steady-state for 30 min (application time). Toxic aerosol was exhausted over a closed system. Trocars were retracted. PIPAC was repeated two to five times at various time intervals. Occupational health safety aspects are described elsewhere. ${ }^{12}$

\section{Safety and Efficacy Assessments}

Assessments for patient safety and tolerability were performed from days 1-5 after treatment and included medical examination and routine laboratory measures. Adverse effects were graded according to the NCI Criteria for Adverse Events (CTCAE). ${ }^{13}$ Tumor response was

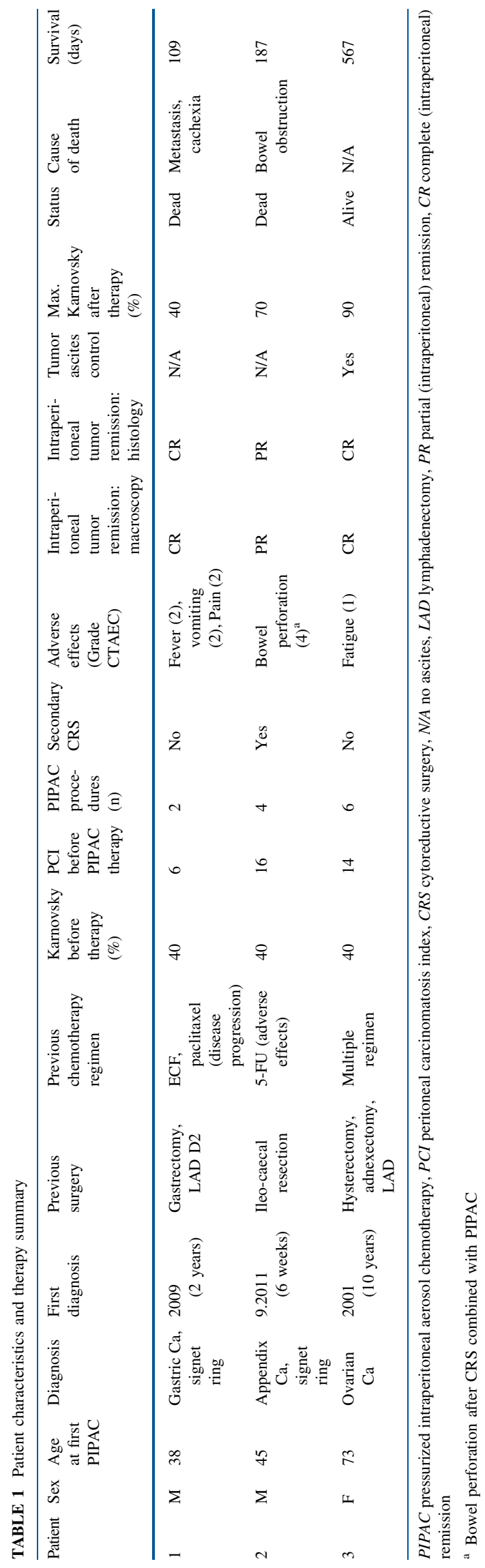


FIG. 1 Pressurized intraperitoneal aerosol chemotherapy (PIPAC). The procedure is performed in an operating room equipped with laminar air-flow and is remotecontrolled. In a first step, a normothermic capnoperitoneum is established with a pressure of $12 \mathrm{mmHg}$ at body temperature. A chemotherapy solution (about $10 \%$ of a normal systemic dose) is nebulized with a micropump into the tightly closed abdominal cavity, and maintained for $30 \mathrm{~min}$. The toxic aerosol is then exhausted through a closed system and released into the external environment

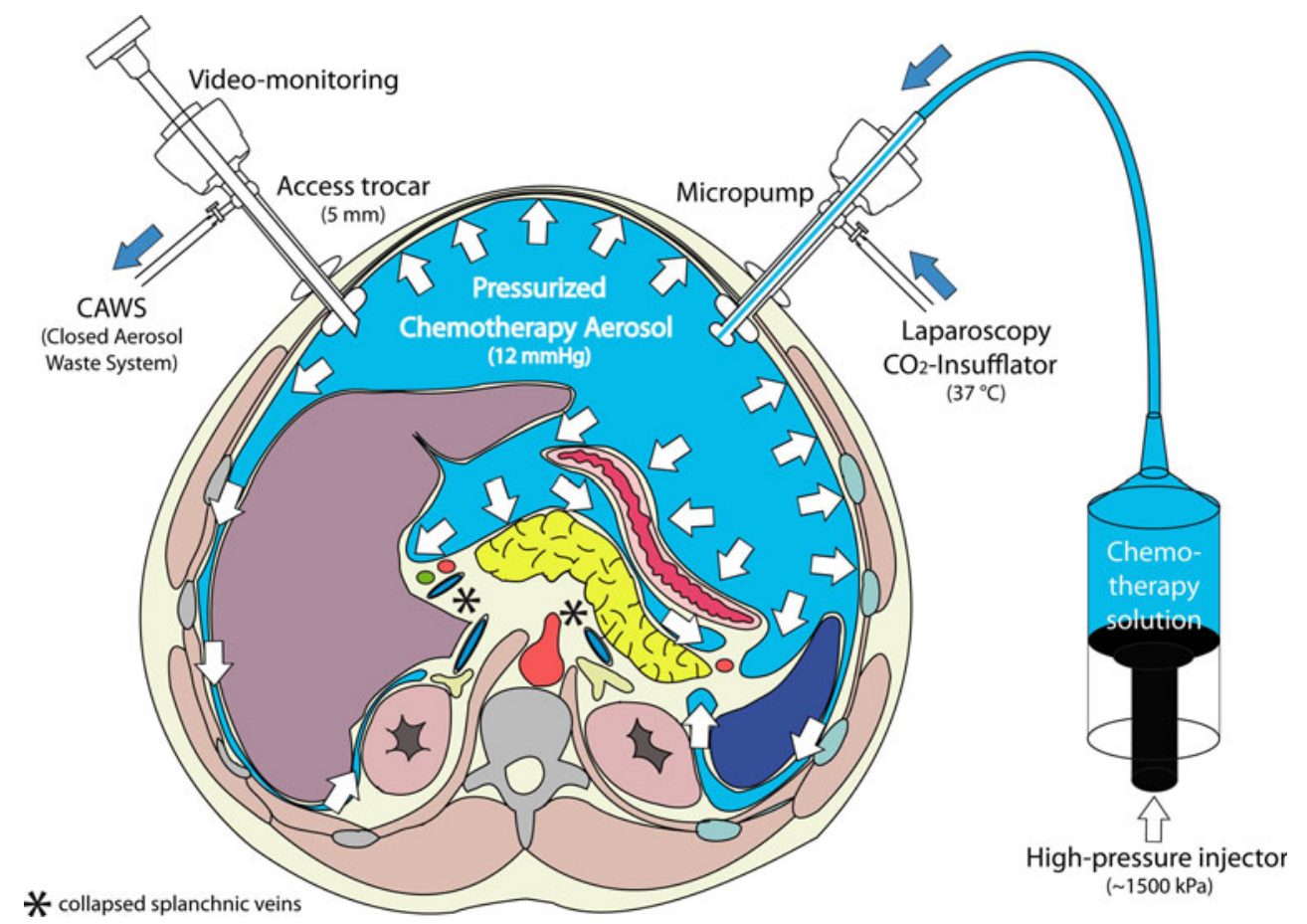

assessed by laparoscopy with macroscopic assessment and histology, as part of the next PIPAC cycle. Patients were followed up for analysis until June 20, 2013 or until death.

\section{Histology}

Biopsies were analyzed for possible tumor response by conventional HE microscopy.

\section{Clinical Pharmacology}

Analysis is based on eight PIPAC in three patients. Blood samples were drawn before, during, and up to $12 \mathrm{~h}$ after start of PIPAC. At the end of PIPAC, biopsies from peritoneal tissue and tumor nodules were snap-frozen. Doxorubicin plasma levels were determined by UHPLCMS/MS using [13C2H3]-doxorubicin as internal standard. Pharmacokinetics parameters were derived by non-compartmental analysis (WinNonLin 6.3, Pharsight, Cary, NC, USA). The area under the plasma concentration-time curve (AUC) was calculated by the linear trapezoidal rule.

\section{RESULTS}

\section{Patient 1}

A 45-year-old male patient was operated because of acute bowel obstruction due to PC from signet-ring appendiceal cancer. Postoperative high-dose chemotherapy
(5-FU) was interrupted due to acute heart failure. After recovery, Karnovsky index was $40 \%$. First PIPAC was performed, showing a PCI of 16 (Fig. 2a1, b1). Four weeks later, second PIPAC showed stable disease. Four weeks later, 3rd PIPAC showed hard, glassy nodules (a2), histology showed regressive changes with nodular sclerosis (b2). Six weeks later, small bowel nodules were regressive (a3), histology showed regressive changes with $60 \%$ vital cells and large devitalized areas (b3). Complete CRS was performed, and fourth PIPAC administered. A postoperative bowel perforation required surgical revision. After recovery, the patient developed bowel obstruction and tumor progression was confirmed by laparotomy. He died 187 days after first PIPAC.

\section{Patient 2}

A 38-year-old male patient with a 2-year history of signet-ring gastric cancer, gastrectomy, and two chemotherapy lines presented with tumor progression and endstage disease requiring parenteral nutrition. Karnovsky index was $40 \%$. A PCI of 6 was documented at first PIPAC (Supplementary material 1, a1). Four weeks later, during the second PIPAC, macroscopy showed complete remission (a2), and multiple biopsies confirmed absence of tumor cells (b2). Eight weeks later, the patient developed liver and bone metastases and died 109 days after the first PIPAC. Two weeks before death, abdomen CT showed no evidence of PC. 


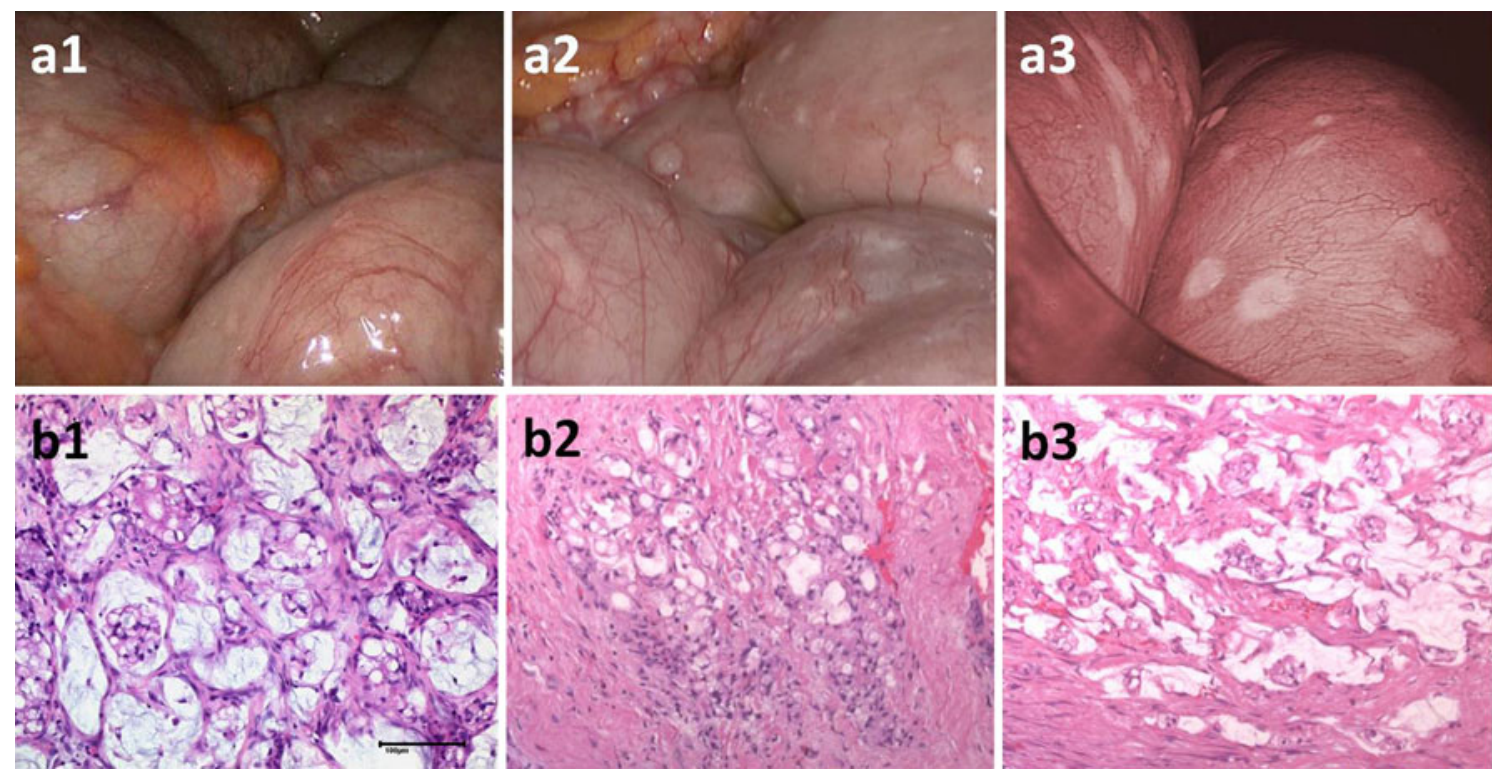

FIG. 2 Macroscopical and histological response after PIPAC. Fortyfive year male patient with diffuse peritoneal carcinomatosis $(\mathrm{PCI}=16)$ from a signet-ring cells appendiceal cancer with inaugural small bowel obstruction. Macroscopy before (a1), after 1st (a2) and

\section{Patient 3}

A 73-year-old female patient with a 10-year history of ovarian cancer, surgery, and multiple chemotherapy regimens presented with tumor progression and hemorrhagic ascites. Karnovsky index was $40 \%$. At first PIPAC, a PCI of 14 was documented and 2.51 ascites removed (Fig. 3a1, b1). At second PIPAC after 4 weeks, PC nodules were hard and glassy, and ascites volume dropped to 11 . Histology showed no regression (not shown). Six weeks later at the third PIPAC, ascites was $<500 \mathrm{ml}$ (a2), and histology showed partial tumor response with fibrotic reaction (b2). Karnoswky index was $90 \%$. Eight months after first PIPAC, fourth PIPAC showed complete remission (a3), and multiple biopsies showed apoptotic inflammatory cells (b3) but no tumor. After 15 months of follow-up, the asymptomatic patient underwent control laparoscopy; tumor was documented in two of five peritoneal biopsies, so fifth PIPAC was applied. Six weeks later, a single, 6-cm, large tumor node was resected. All other biopsies were negative; sixth PIPAC was applied. After 567 days, the patient is alive with an excellent quality of life.

\section{Safety}

All 11 PIPAC procedures were technically easy to perform. For PIPAC alone, mean operating time was $93 \pm 13$ min. No intraoperative complication was noted. PIPAC alone was very well tolerated, no adverse effect $>2$ CTCAE was noted (Table 1). After PIPAC alone, patients after 3rd PIPAC (a3) showing regression of small bowel PC nodules. Histology shows vital tumor before PIPAC (b1), inflammatory reaction with nodular sclerosis after 1st PIPAC (b2) and large areas of devitalized tumor after 3rd PIPAC (b3). Scale bar $100 \mu \mathrm{m}$

were discharged from hospital 2-5 days after treatment. In two instances, PIPAC was combined with another operation (small-bowel resection, complete cytoreductive surgery): in the latter case, a postoperative bowel perforation (see Patient 1 above) required surgical revision.

\section{Clinical Pharmacology}

The plasma concentration-time curve fitted best to a two-compartment model with first-order absorption. Peak doxorubicin plasma concentrations were low (4.0-6.2 ng/ $\mathrm{ml}$ ) and were reached with the end of nebulisation. Doxorubicin was eliminated from the body with a clearance $(\mathrm{Cl} / \mathrm{F})$ of $2.6-6.0 \mathrm{ml} / \mathrm{min}$. Half-lives and AUCs ranged from 86 to $468 \mathrm{~min}$ and 415 to $915 \mathrm{ng} / \mathrm{ml} \mathrm{min}$, respectively (Fig. 4a). Tissue concentration of doxorubicin was high (mean $1.7 \mu \mathrm{g} / \mathrm{g}$ ) and variable (SD $\pm 1.45 \mu \mathrm{g} / \mathrm{g}$ ). In tumor nodules, fluorescence microscopy showed nuclear presence of doxorubicin up to $500 \mu \mathrm{m}$ depth (Fig. 4b) and throughout the whole peritoneal layer into the properitoneal fatty tissue $(>600 \mu \mathrm{m}$, Supplementary material 2). Highest concentration was achieved within 100-200 $\mu \mathrm{m}$ from the surface.

\section{DISCUSSION}

To our knowledge, this is the first report of successful application of chemotherapy as a pressurized aerosol within the abdomen of human patients. It has been predicted that innovative concepts overcoming pharmacologic 

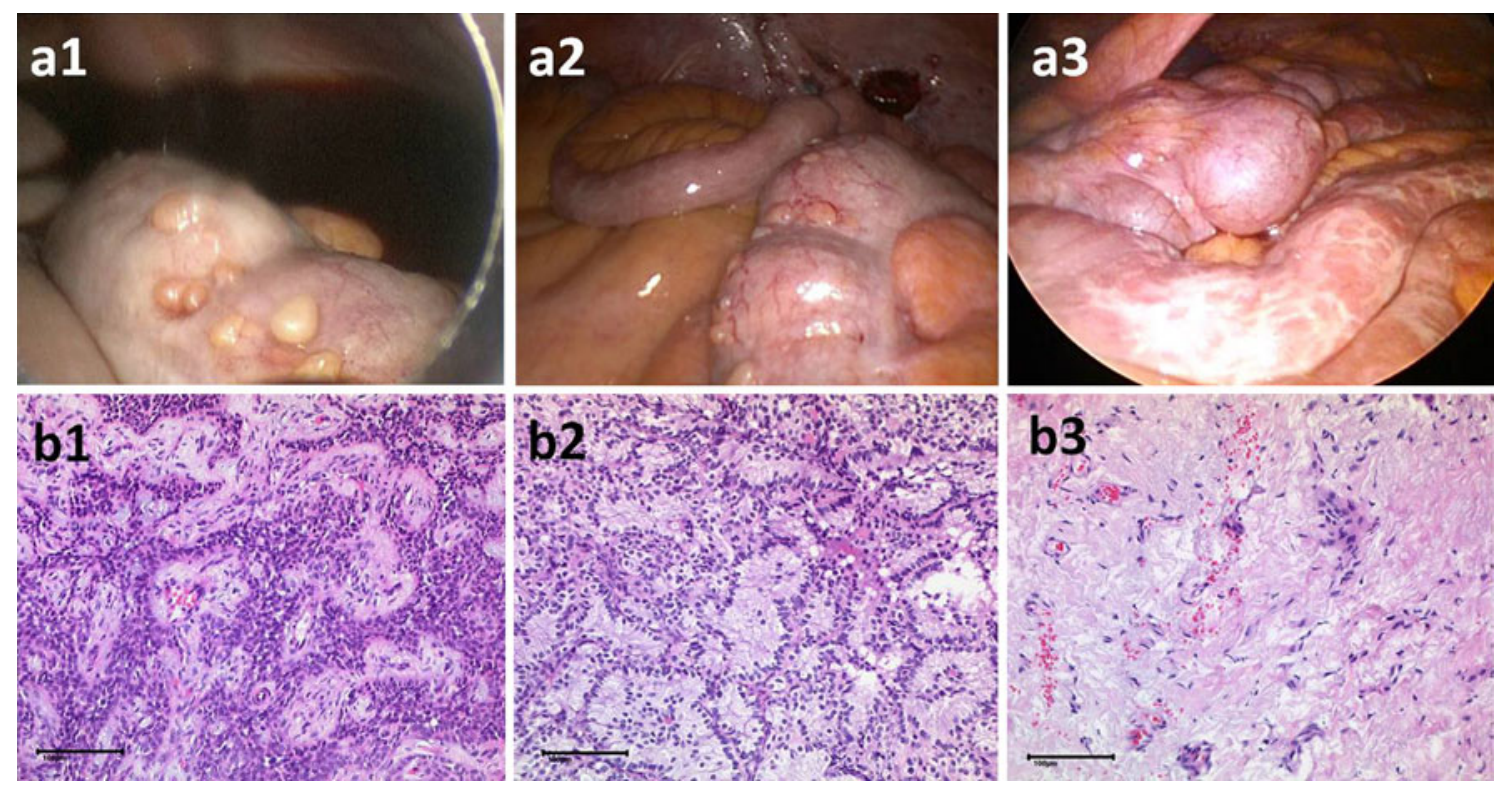

FIG. 3 73-year-old patient (patient 3) with therapy-resistant peritoneal carcinomatosis and malignant ascites of ovarian origin. Shown is the macroscopic (upper fig) and histological (HE staining, lower fig) appearance before (left column), after PIPAC (middle column) and at

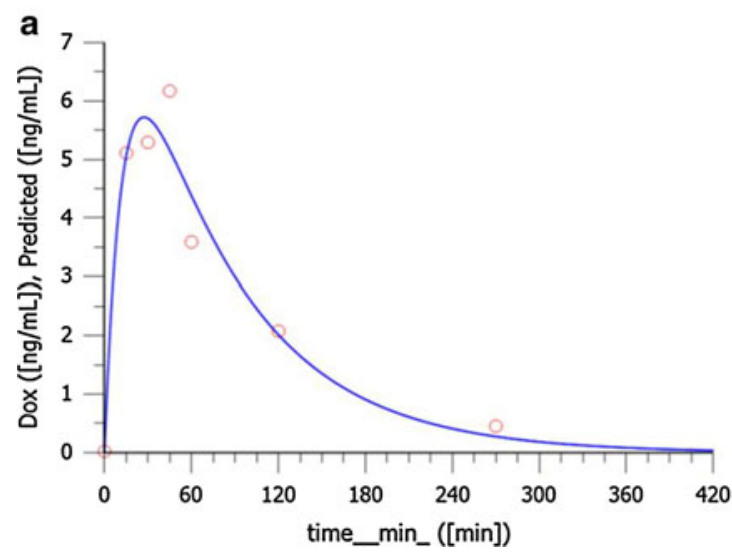

FIG. 4 Local and systemic doxorubicin exposure during PIPAC. Local disposition is high with $1.70 \pm 1.45 \mu \mathrm{g} / \mathrm{g}$. In a fluorescence microscopy shows a nuclear presence of doxorubicin up to $500 \mu \mathrm{m}$ depth. Red doxorubicin. Green picogreen nuclear counterstaining. Scale bar $100 \mu \mathrm{m}$. b Shows a typical pharmacokinetic profile in

limitations of IPC could improve, perhaps dramatically, its efficacy. ${ }^{5}$ A superior dose-response ratio after PIPAC could be expected from preclinical data. In the human patient, plasma concentration-time profile analysis now confirms such superior ratio between dose, systemic, and local drug concentration: PIPAC required only $1 / 10$ of the doxorubicin dose to achieve higher tumor concentrations $(0.03-4.1 \mu \mathrm{mol} / \mathrm{g})$ as reported for HIPEC $(0.02 \mu \mathrm{mol} / \mathrm{g}){ }^{15}$ In contrast, systemic availability of doxorubicin after PIPAC and HIPEC were equal as indicated by the approximately ten times lower maximal plasma concentration after PIPAC. later stages of follow-up (right column). a, b Show partial and then complete macroscopic and microscopic tumor remission after repeated PIPAC. Scale bar $100 \mu \mathrm{m}$

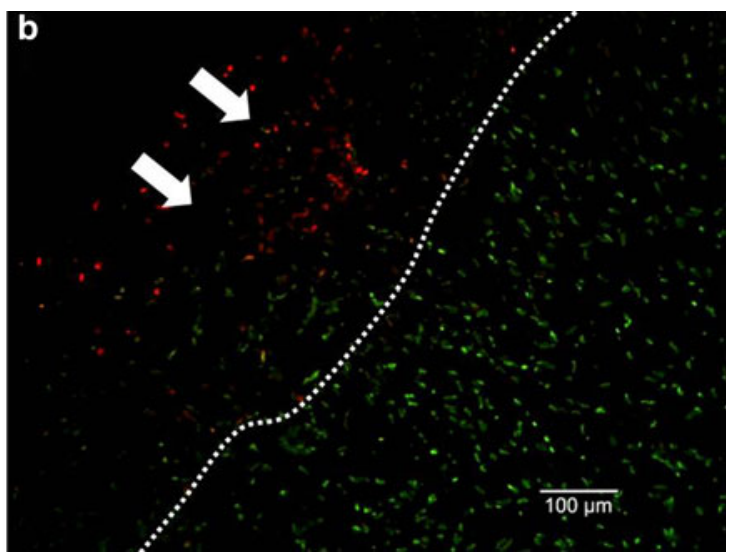

peripheral venous blood after PIPAC with doxorubicin $1.5 \mathrm{mg} / \mathrm{m}^{2}$ body surface for $30 \mathrm{~min}$ at an intraabdominal pressure of $12 \mathrm{mmHg}$. Peak doxorubicin plasma concentrations were low $(4.0-6.2 \mathrm{ng} / \mathrm{ml})$. Line predicted profile. Dots experimental values

We and others have reported that increasing intraperitoneal pressure enhanced particularly the uptake of drugs into the tumor, resulting in a higher local disposition. ${ }^{9,10,15,16}$ After PIPAC, doxorubicin was not only detected in significant concentrations in PC nodules, but nuclear staining was demonstrated throughout the peritoneum, up to deeply into the retroperitoneal fatty tissue. Another explanation for superior local disposition could be the high drug concentration in the aerosol. Although used in only $1 / 10$ of the total dose, doxorubicin concentration in the aerosol $(52 \mu \mathrm{M})$ is three times higher as in the intraperitoneal fluid usually used in HIPEC $(18 \mu \mathrm{M})$ without 
impairing tolerability, which was reported after applying higher concentrations of IPC. ${ }^{15,17}$

Tumor response was observed in all three cases after PIPAC, as a consequence of the well-documented antitumor activity of doxorubicin and cisplatin and the superior local disposition. However, we were surprised by the extent of macroscopic and microscopic response in these multidrug-resistant tumors. We documented a complete remission of $\mathrm{PC}$ in two patients, which was indeed unexpected.

At this stage, it would be clearly premature to claim that combined PIPAC with cisplatin and doxorubicin improves survival in advanced PC. However, in our three patients with multiresistant tumors, low performance index, and very limited life expectancy, we observe a mean survival of more than 288 days. Remarkably, 567 days after her first PIPAC patient 3 is still alive.

In sharp contrast to HIPEC, PIPAC was very well tolerated and the only severe adverse effect observed was a bowel perforation after CRS (Table 1). Otherwise, postoperative courses were uneventful, with early hospital discharge.

PIPAC might create synergies with SC. Liver and renal tests showed neither acute nor cumulative toxicity after PIPAC, which appears reasonable bearing in mind the $90 \%$ dose reduction compared with conventional SC. ${ }^{19}$ Moreover, PIPAC permits repeated cycles of IPC and therefore might allow effective regimen combining SC and PIPAC. Importantly, repeated laparoscopy enables objective staging, assessment of therapeutic response, and adaptation of further therapy accordingly, which was barely possible until now. Finally, considering that all three patients were in poor physical condition with a low performance index, PIPAC might allow therapy in polymorbid patients-when SC is contraindicated.

We observed tumor regression even in platin-resistant tumors, after application of cisplatin and doxorubicin. This is not surprising since drug effect is usually dose-dependent. PIPAC might become an alternative therapy for platin-resistant tumors, in particular in women with ovarian cancer where tumor progression is diagnosed after first-line therapy with carboplatin-Taxol. Repeated intraoperative analysis of the environmental air showed that PIPAC is safe for staff and meets the requirements of the German working safety regulations. ${ }^{12}$

\section{CONCLUSIONS}

These early data are promising: PIPAC can induce remission in end-stage, therapy-resistant $\mathrm{PC}$, and first safety data are encouraging. PIPAC is well tolerated, a decisive feature in patients with limited life expectancy. By requiring only $10 \%$ of the dose of conventional IPC, PIPAC shows an excellent local distribution with low systemic exposure. Furthermore, PIPAC permits repeated cycles of IPC as well as objective tumor staging and response assessment. PIPAC is easy to use. PIPAC is complying with EC occupational safety regulations. The potential of this generic technology for a variety of indications and drugs has now to be determined with adequate studies.

ACKNOWLEDGMENT This study was supported by Robert Bosch Foundation (Stuttgart, Germany) and the Federal Ministry for Education and Research (BMBF, Berlin, Germany) Grant \#0316186D.

OPEN ACCESS This article is distributed under the terms of the Creative Commons Attribution License which permits any use, distribution, and reproduction in any medium, provided the original author(s) and the source are credited.

\section{REFERENCES}

1. Griffiths RW, Zee YK, Evans S, Mitchell CL, Kumaran GC, Welch RS, Jayson GC, Clamp AR, Hasan J. Outcomes after multiple lines of chemotherapy for platinum-resistant epithelial cancers of the ovary, peritoneum, and fallopian tube. Int $J$ Gynecol Cancer. 2011;21(1):58-65.

2. Minchinton AI, Tannock IF. Drug penetration in solid tumours. Nat Rev Cancer. 2006;6(8):583-92. Review.

3. Macrì A, Fortugno A, Saladino E. Rationale and techniques of CRS and peritoneal chemohyperthermia. World J Gastrointest Oncol. 2011;3(12):169-74.

4. Sugarbaker PH, Ryan DP. Cytoreductive surgery plus hyperthermic perioperative chemotherapy to treat peritoneal metastases from colorectal cancer: standard of care or an experimental approach? Lancet Oncol. 2012;13(8):e362-9.

5. Dedrick RL, Flessner MF. Pharmacokinetic problems in peritoneal drug administration: tissue penetration and surface exposure. J Natl Cancer Inst. 1997;89:480-7.

6. Elias D, Gilly F, Boutitie F, Quenet F, Bereder JM, Mansvelt B, Lorimier G, Dubè P, Glehen O. Peritoneal colorectal carcinomatosis treated with surgery and perioperative intraperitoneal chemotherapy: retrospective analysis of 523 patients from a multicentric French study. J Clin Oncol. 2010;28(1):63-8.

7. Reymond MA, Hu B, Garcia A, et al. Feasibility of therapeutic pneumoperitoneum in a large animal model using a microvaporisator. Surg Endosc. 2000;14:51-5.

8. Heldin $\mathrm{CH}$, Rubin K, Pietras K, Ostman A. High interstitial fluid pressure - an obstacle in cancer therapy. Nat Rev Cancer. 2004;4(10):806-13. Review.

9. Solaß W, Hetzel A, Nadiradze G, Sagynaliev E, Reymond MA. Description of a novel approach for intraperitoneal drug delivery and the related device. Surg Endosc. 2012;26(7):1849-55.

10. Solass W, Herbette A, Schwarz T, Hetzel A, Sun JS, Dutreix M, Reymond MA. Therapeutic approach of human peritoneal carcinomatosis with Dbait in combination capnoperitoneum: proof of concept. Surg Endosc. 2012;26(3):847-52.

11. Elias D, Souadka A, Fayard F, Mauguen A, Dumont F, Honore C, Goere D. Variation in the peritoneal cancer index scores between surgeons and according to when they are determined (before or after cytoreductive surgery). Eur J Surg Oncol. 2012;38(6):503-8.

12. Solaß W, Giger U, Borgstedt U, Zieren J, Reymond MA. Pressurized intraperitoneal aerosol chemotherapy (PIPAC): occupational 
health and safety aspects. Ann Surg Oncol. 2013 Jun 14 [Epub ahead of print].

13. http://ctep.cancer.gov/protocolDevelopment/electronic_applications/ ctc.htm, Accessed 31 Dec 2012.

14. Sugarbaker PH, Van der Speeten K, Anthony Stuart O, Chang D. Impact of surgical and clinical factors on the pharmacology of intraperitoneal doxorubicin in 145 patients with peritoneal carcinomatosis. Eur J Surg Oncol. 2011;37(8):719-26.

15. Jacquet $P$, Stuart OA, Chang D, Sugarbaker PH. Effects of intraabdominal pressure on pharmacokinetics and tissue distribution of doxorubicin after intraperitoneal administration. Anticancer Drugs. 1996;7(5):596-603.

16. Esquis P, Consolo D, Magnin G, Pointaire P, Moretto P, Ynsa MD, Beltramo JL, Drogoul C, Simonet M, Benoit L, Rat P, Chauffert B.
High intra-abdominal pressure enhances the penetration and antitumor effect of intraperitoneal cisplatin on experimental peritoneal carcinomatosis. Ann Surg. 2006;244(1):106-12.

17. Ozols RF, Young RC, Speyer JL, Sugarbaker PH, Greene R, Jenkins J, Myers CE. Phase I and pharmacological studies of adriamycin administered intraperitoneally to patients with ovarian cancer. Cancer Res. 1982;42(10):4265-9.

18. Markman M. Intraperitoneal antineoplastic drug delivery: rationale and results. Lancet Oncol. 2003;4:277-83.

19. Blanco A, Giger U, Solass W, Cruciger O, Zieren J, Reymond MA. Renal and hepatic toxicities after pressurized intraperitoneal aerosol chemotherapy (PIPAC). Ann Surg Oncol. 2013;20(7): 2311-6. 